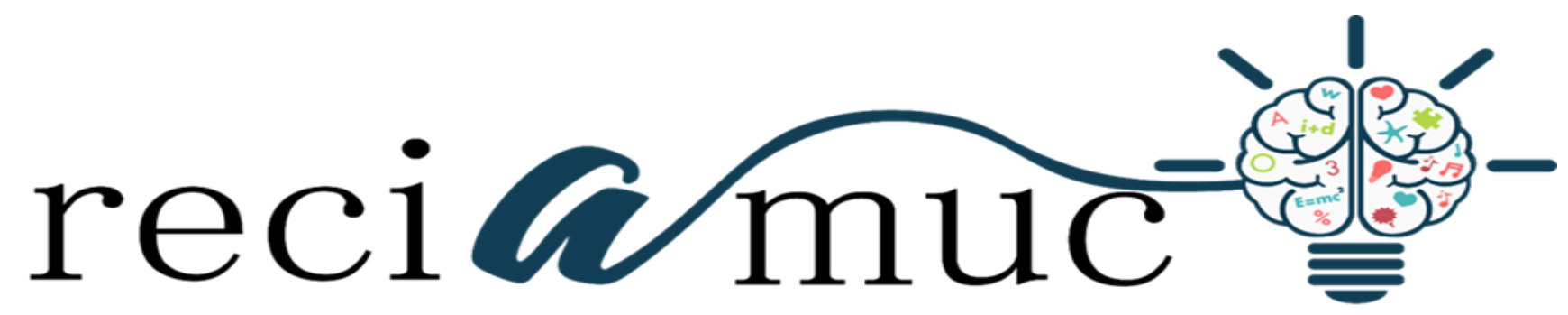

Revista científica de investigación actualización del mundo de las ciencias

$$
\text { Celia María Pulgarin Fernández a }
$$

Generalidades de la medicina crítica o intensivista

Generalities of critical or intensivist medicine

Revista Científica de Investigación actualización del mundo de las Ciencias. Vol. 3 núm., 2, abril, ISSN: 2588-0748, 2018, pp. 376-394

DOI: 10.26820/reciamuc/3.(2).abril.2019.376-394

URL: http://reciamuc.com/index.php/RECIAMUC/article/view/345

Código UNESCO: 3205 Medicina Interna

Tipo de Investigación: Artículo de Revisión

(C) RECIAMUC; Editorial Saberes del Conocimiento, 2019

Recibido: $15 / 01 / 2019$

Aceptado: 07/02/2019

Publicado: 01/04/2019

Correspondencia: celita mtz@hotmail.com

a. Médica General; Especialista en Medicina Crítica y Terapia Intensiva; Investigador Independiente; Guayaquil, Ecuador; celita_mtz@hotmail.com 


\section{Generalidades de la medicina crítica o intensivista}

Vol. 3, núm. 2., (2019)

Celia María Pulgarin Fernández

\section{RESUMEN}

Se define la Medicina Critica (MC) o Medicina Intensiva (M.I.) como aquella parte de la Medicina, que se ocupa de los pacientes con una patología que haya alcanzado un nivel de severidad tal, que suponga un peligro vital, actual o potencial, susceptible de recuperabilidad. Un conjunto de procedimientos invasivos, diagnósticos y terapéuticos, son esenciales para el manejo de los pacientes críticos. Es reconocido que los sistemas de soporte vital alteran los mecanismos normales de defensa del huésped, afectando a un paciente con una respuesta inmune ya deteriorada por su enfermedad de base. Más de un tercio de los pacientes admitidos a las unidades de cuidados intensivos experimentan eventos adversos inesperados, siendo las infecciones nosocomiales una de las complicaciones más frecuentes. La metodología de la investigación es de tipo bibliográfica. Las conclusiones más relevantes es que se confirman todos los riesgos a los que están sometidos los pacientes críticos que deben estar hospitalizados en unidades de cuidados intensivos, al contraer infecciones, bacterias, enfermedades como neumonía, entre otras. Es por ello que siempre debe haber un monitoreo importante dentro de las áreas críticas y que se cumplan los protocolos higiénicos, de seguridad y médicos para evitar las complicaciones de pacientes críticos, al final la medicina critica lo que busca es la recuperación exitosa del paciente.

Palabras clave: UCI; Critica; Medicina; Infecciones; Protocolos. 


\title{
Generalidades de la medicina crítica o intensivista
}

Vol. 3, núm. 2., (2019)

Celia María Pulgarin Fernández

\begin{abstract}
Critical Medicine (CM) or Intensive Medicine (MI) is defined as that part of Medicine, which deals with patients with a pathology that has reached a level of severity, which poses a vital, current or potential danger, susceptible of recoverability. A set of invasive procedures, diagnostic and therapeutic, are essential for the management of critical patients. It is recognized that life support systems alter the normal defense mechanisms of the host, affecting a patient with an immune response already impaired by their underlying disease. More than a third of patients admitted to intensive care units experience unexpected adverse events, with nosocomial infections being one of the most frequent complications. The research methodology is bibliographic. The most relevant conclusions are that all the risks to which critical patients who are hospitalized in intensive care units are confirmed, when they get infections, bacteria, diseases such as pneumonia, among others, are confirmed. That is why there must always be an important monitoring within the critical areas and that the hygienic, safety and medical protocols are met to avoid the complications of critical patients, in the end the critical medicine that seeks is the successful recovery of the patient.
\end{abstract}

Key words: ICU; Criticism; Medicine; Infections; Protocols. 


\section{Introducción.}

Se define la Medicina Critica (MC) o Medicina Intensiva (M.I.) como aquella parte de la Medicina, que se ocupa de los pacientes con una patología que haya alcanzado un nivel de severidad tal, que suponga un peligro vital, actual o potencial, susceptible de recuperabilidad. El concepto actual de terapéutica intensiva o Terapia intensiva, comprende la aplicación sistemática de las múltiples posibilidades terapéuticas modernas, que se utilizan en situaciones de peligro para la vida, lo que supone la sustitución temporal de las funciones orgánicas alteradas o suprimidas, sin abandonar por ello el tratamiento simultaneo de la enfermedad de base, que ha dado lugar a estos trastornos y teniéndose en cuenta que tales medidas y al final de la terapéutica, proporcionaran una buena calidad de vida para el futuro (Vera Carrasco, 2015, pág. 77).

La historia de la Medicina Intensiva es reciente comparada con la de otras disciplinas médicas, y su aparición y desarrollo han venido determinados tanto por la necesidad de atender adecuadamente a los pacientes críticos como por la disponibilidad de recursos para hacerlo. En cada país el camino tomado ha venido marcado por sus propias circunstancias, y a menudo se ha encontrado con estructuras previas y rigideces que han condicionado su desarrollo (Palencia Herrejón, González Díaz, \& Mancebo Cortés, 2011, pág. 233).

Son ámbitos de actuación de la medicina crítica las Unidades de Terapia Intensiva (UTI) o Cuidado intensivo, tanto los polivalentes como los dedicados a una sola especialidad, médica o quirúrgica, y otras áreas del Sistema de Salud donde haya pacientes gravemente enfermos que requieran una atención integral. Como consecuencia de la progresiva demanda social que exige 


\section{Generalidades de la medicina crítica o intensivista}

Vol. 3, núm. 2., (2019)

Celia María Pulgarin Fernández

cada vez mayores niveles de asistencia, y del desarrollo profesional que permite asistir a los pacientes por encima de los límites convencionales, la medicina crítica representa la adquisición de competencias que son la suma de conocimientos, habilidades y actitudes que significan el último escalón asistencial de un sistema de progresiva atención a los pacientes gravemente enfermos (Vera Carrasco, Programas académicos de la residencia médica en medicina crítica y terapia intensiva, 2012).

En principio, la terapia intensiva basa su atención en el reconocimiento oportuno y preciso de desviaciones de variables fisiológicas y bioquímicas en el paciente enfermo de gravedad. Partiendo de lo anterior, se procede a normalizar dichas variables con el fin de recuperar al enfermo. Sin embargo, surge la interrogante: ¿es lo correcto en todos los casos aplicar esta estrategia de normalización o corrección de lo que se encuentra fuera de la normalidad, o podría ser contraproducente? Esta nueva forma de interpretar las manifestaciones de la enfermedad puede tener repercusiones en su tratamiento y una utilización más racional de los recursos (PadillaCuadra, 2018, pág. 8).

Un conjunto de procedimientos invasivos, diagnósticos y terapéuticos, son esenciales para el manejo de los pacientes críticos. Es reconocido que los sistemas de soporte vital alteran los mecanismos normales de defensa del huésped, afectando a un paciente con una respuesta inmune ya deteriorada por su enfermedad de base. Más de un tercio de los pacientes admitidos a las unidades de cuidados intensivos experimentan eventos adversos inesperados, siendo las infecciones nosocomiales una de las complicaciones más frecuentes. Las infecciones nosocomiales adquiridas en UTI aumentan la morbilidad, el tiempo de estadía y los costos hospitalarios. En adición, la neumonía asociada al ventilador y las infecciones hematógenas adquiridas en UTI 


\section{Generalidades de la medicina crítica o intensivista}

Vol. 3, núm. 2., (2019)

Celia María Pulgarin Fernández

tienen una mortalidad atribuible variable entre el 10 y el $35 \%$ en diferentes poblaciones. Afortunadamente, los estudios sistemáticos destinados a reconocer los factores de riesgo de la infección nosocomial, la vigilancia de las infecciones y la adherencia a guías prácticas de prevención han sido efectivos para reducir el riesgo de los pacientes admitidos en terapia intensiva (Pittet \& Harbarth, 2006).

Entonces, la medicina intensiva es una de las áreas más importantes de la medicina, ya que en ella puede darse un agravamiento y posterior muerte del paciente o su recuperación que le permita su salida de la unidad de cuidados intensivos. Los pacientes en esta área de la medicina no solo están en riesgo por el padecimiento que tengan, el cual los llevo a esas instancias, sino a otras aspectos que no son menos importantes como las infecciones, bacterias, malos cuidados de enfermería y malas decisiones médicas.

\section{Metodología}

La metodología de la investigación es de tipo bibliográfica, la misma se centra en un abordaje de aspectos generales sobre la medicina critica o intensiva, para ello se ha recurrido a diferentes medios electrónicos para la obtención de la información de diferentes autores que ya han generalizado sobre estos temas y otros que han sido más específicos y que han ayudado a la construcción de esta investigación.

\section{Resultados}

Estrategias de normalización 


\section{Generalidades de la medicina crítica o intensivista}

Vol. 3, núm. 2., (2019)

Celia María Pulgarin Fernández

Control de glicemia: la insulinoterapia intensiva, no es recomendable para el control de la glicemia en vista de que aunque puede llevarla a números normales y en algunos casos reducir la mortalidad. Puede producir en los pacientes hipoglicemias lo que puede generar más bien todo lo contrario. Esto debido a casos demostrados donde se reducía los niveles de mortalidad del 8 al 4,6\% llevando la glicemia con insulinoterapia a valores normales de 80-110 mg/dl. Sin embargo estudios de NICESUGAR (The Normoglycemia in Intensive Care Evaluation Survival Using Glucose Algorithm Regulation Study), han desmontado esta práctica, donde más bien infieren que los procesos de stress sometidos en las unidades de cuidados intensivos (UCI) pueden liberar en el paciente catecolaminas y cortisol que conducen a una hiperglicemia, que puede llevar a sobrevivir al paciente.

Falla renal: Estudios de Acker et al, demostraron que controlar las fallas renales por medio de tiroxina aumento la mortalidad de un 13 a un 46\%. Pacientes con el llamado síndrome de eutiroideo enfermo, que se caracteriza por niveles disminuidos de T3 en pacientes críticos, no debe tratarse con suplemento hormonal. En estados de suma gravedad una hormona estimula el catabolismo.

\section{Infecciones en las UCI}

La infección nosocomial endémica más común en UTI es la que afecta al tracto respiratorio inferior (40\%), seguida por la del tracto urinario (24\%) y la bacteriemia (11\%). La elevada incidencia de infecciones pulmonares en relación con otros sitios de infección es particular de las unidades de cuidados críticos, donde los pacientes con frecuencia requieren intubación endotraqueal 


\section{Generalidades de la medicina crítica o intensivista}

Vol. 3, núm. 2., (2019)

Celia María Pulgarin Fernández

Aunque menos del $10 \%$ de los pacientes hospitalizados son tratados en terapia intensiva, muchos brotes de infecciones nosocomiales ocurren en estas unidades. En la University of Virginia Hospital, 10 de 11 brotes epidémicos identificados entre 1978 y 1982 ocurrieron en UTI, y ocho de ellos involucraron infecciones hematógenas. Similar a los brotes de infecciones hematógenas polimicrobianas descriptas por Ponce de León y colaboradores, aquéllos estuvieron frecuentemente relacionados con un empleo inadecuado de la técnica o con una falta de observación de las reglas de control de infecciones. Otras epidemias fueron asociadas con tipos específicos de bacterias o estuvieron relacionadas con reservorios inanimados contaminados. En efecto, las epidemias causadas por organismos inusuales, tales como el Acinetobacter spp., generalmente se asocian con un equipamiento contaminado o con cambios en el medio ambiente.

La literatura reciente está plena de informes sobre la falta de observancia de las técnicas de control de infecciones, lo que conduce a epidemias de infecciones nosocomiales en UTI. Los autores recientemente publicaron una revisión extensa de más de 50 episodios de infección nosocomial que ocurrieron en UTIs entre 1983 y 1995 (Harbarth S., Pittet D., 1996). En conjunto, aproximadamente el $40 \%$ de los mismos fueron causados por bacilos Gram negativos y un tercio por bacterias Gram positivas. Los patógenos más frecuentemente aislados fueron el Staphylococcus aureus meticilino resistente, Klebsiella spp y Pseudomonas spp (Pittet \& Harbarth, 2006).

La influencia y el impacto de los antibióticos se observan en los pacientes que los reciben (respuesta clínica, evolución) y en el ecosistema que rodea al paciente (flora hospitalaria). Este impacto es especialmente visible en los pacientes críticos y en la flora endémica de las unidades de cuidados intensivos. El conjunto de normas y estrategias desarrolladas para mejorar y optimizar 


\section{Generalidades de la medicina crítica o intensivista}

Vol. 3, núm. 2., (2019)

Celia María Pulgarin Fernández

el empleo de los antimicrobianos recibe el nombre de política de antibióticos y consiste en un conjunto de medidas de primer orden en su aplicación en nuestras unidades y también a todos los niveles, hospitalario y en atención primaria (Zaragoza Crespo \& Palomar Martínez, 2011).

Las infecciones comunitarias más graves y frecuentes en la UCI: neumonías comunitarias graves, meningitis, encefalitis e infecciones del tracto urinario, precisan, por su importancia, frecuencia y gravedad, una revisión frecuente y del todo actualizada, como se ha hecho en la puesta al día dedicada a ellas. Con respecto a las neumonías, se hace hincapié en la utilización de las escalas de gravedad para valorar el ingreso en las unidades de críticos, así como en la monitorización evolutiva mediante los marcadores biológicos y en la importancia de instaurar un tratamiento antibiótico precoz adecuado. Se resalta la importancia del Gram de líquido cefalorraquídeo en el diagnóstico etiológico de la meningitis, así como de las técnicas de amplificación por reacción en cadena de polimerasa a tiempo real y de la RM en el diagnóstico etiológico de las encefalitis. Asimismo, se expresa la preocupación por el aumento del porcentaje de cepas de Escherichia coli y Klebsiella pneumoniae productoras de betalactamasas de espectro extendido en nuestro país que nos hace, sin duda, replantear el tratamiento empírico en especial en enfermos graves (Zaragoza Crespo \& Palomar Martínez, 2011).

La Sociedad Española de Medicina Intensiva, Crítica y Unidades Coronarias (SEMICYUC), elaboro unos indicadores de calidad que permiten medir y evaluar la actividad relacionada con la atención del enfermo crítico. 
Tabla 1. Criterios diagnósticos de sepsis

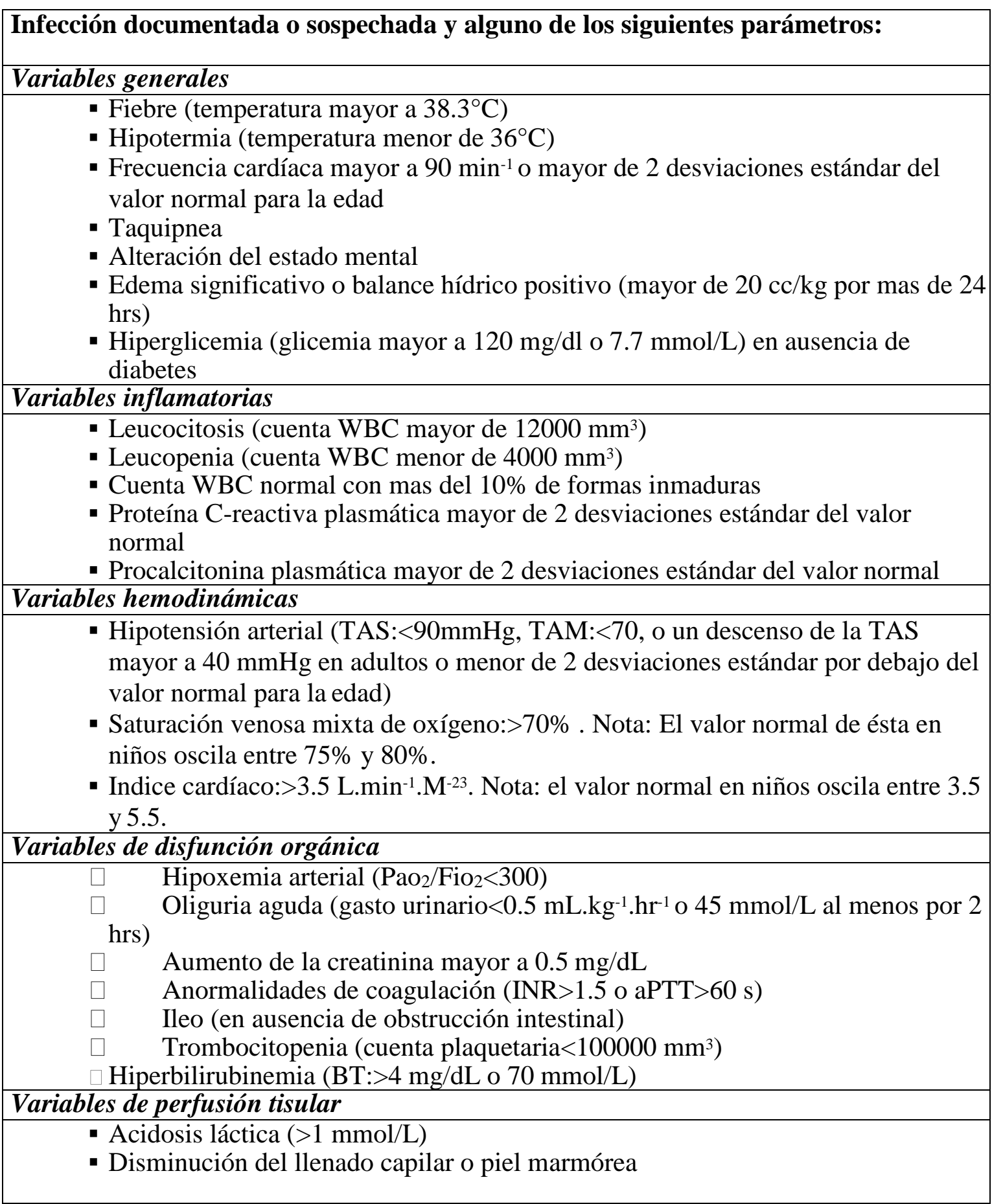

Fuente: (Briceño, 2005). 


\section{Generalidades de la medicina crítica o intensivista}

Vol. 3, núm. 2., (2019)

Celia María Pulgarin Fernández

El control de infecciones tradicional en la UTI

1. Vigilancia para identificar reservorios

a) Pacientes colonizados e infectados

b) Contaminación del medio-ambiente

2. Detener la transmisión entre pacientes

a) Lavado de manos y asepsia adecuados

b) Uso de instrumentos individuales exclusivos

c) Aislar a los pacientes susceptibles: confinamiento, presión negativa, cohortes (pacientes con igual resistencia compartiendo el lugar físico y/o la enfermería)

d) Precaución de barrera (guantes, camisolines) para pacientes colonizados e infectados

e) Eliminar fuentes ambientales de infección; desinfectar el medio-ambiente

f) De ser necesario cerrar la unidad para nuevas internaciones

3. Detener la progresión desde colonización hacia infección

a) Discontinuar factores comprometedores en cuanto sea posible (extubación, extracción de sonda nasogástrica, eliminación de sondas vesicales cuando sea posible; rotar los sitios de punción; adecuada desinfección del respirador y cuidado pulmonar)

4. Modificar factores del huésped

a. Tratar enfermedades subyacentes y complicaciones 


\section{Generalidades de la medicina crítica o intensivista}

Vol. 3, núm. 2., (2019)

Celia María Pulgarin Fernández

b. Controlar el uso de antibióticos (uso de guidelines, restricción, rotación, combinación o cesación)

5. Promover conductas que reduzcan los riesgos de infección-colonización

a) Educación del personal

b) Enfasis en el abordaje del problema por un equipo multi-disciplinario (infectología, personal médico de la UTI, servicios médicos de consulta, microbiología, epidemiología hospitalaria, enfermería) (Luna, Gherardi, Famiglietti, \& Vay, 2001).

\section{Tabla 2. Indicadores de Calidad en el Enfermo Crítico}

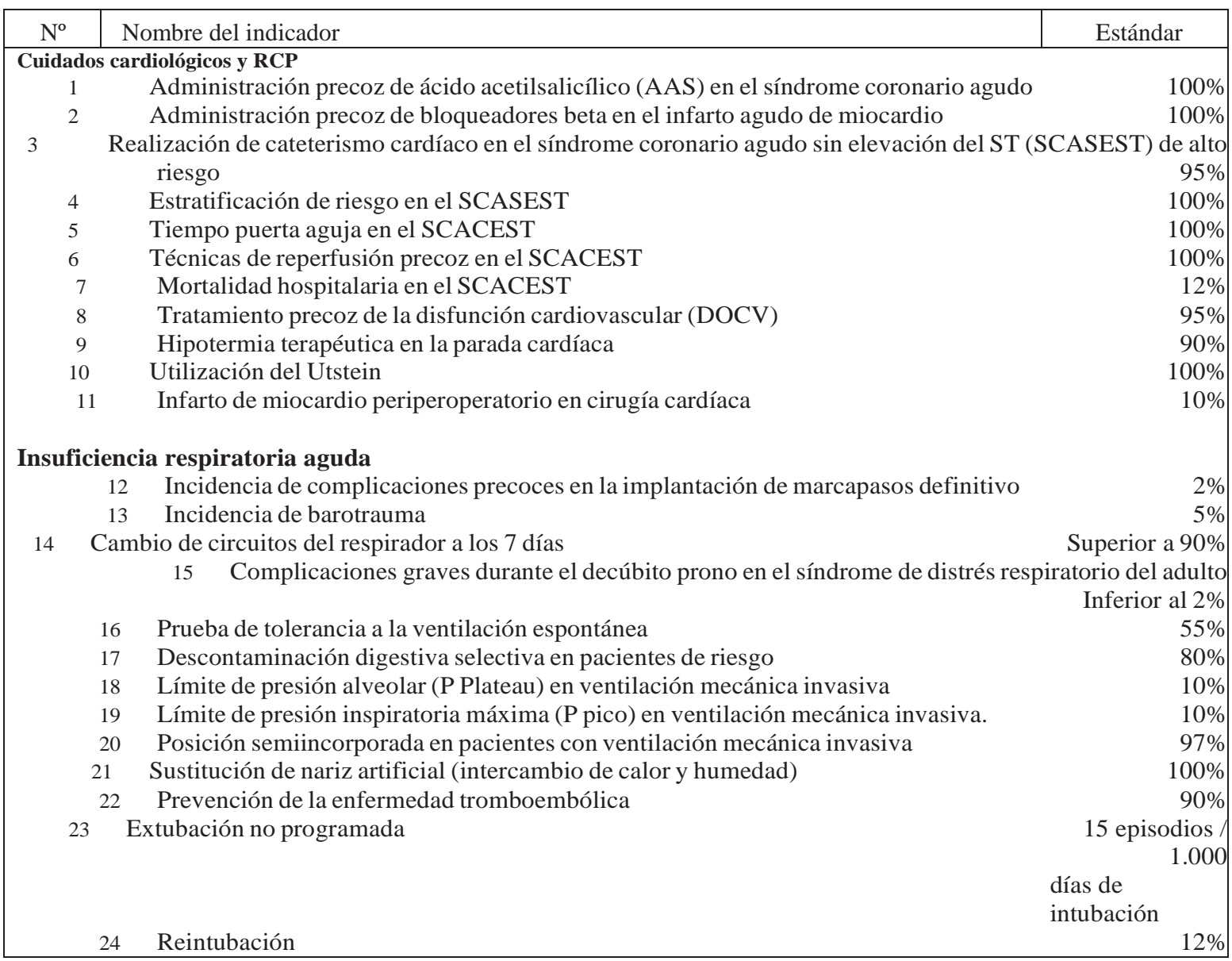




\section{Generalidades de la medicina crítica o intensivista}

Vol. 3, núm. 2., (2019)

Celia María Pulgarin Fernández

25 Instauración precoz de ventilación no invasiva, en la agudización de la enfermedad pulmonar obstructiva

26 Volumen corriente durante la ventilación mecánica invasiva en lesión aguda pulmonar

Neurointensivismo y Traumatología

27 Trauma potencialmente grave atendido en los Servicios de Medicina Intensiva (SMI) 95\%

28 Intubaciones traqueales en el TCE grave con GCS < 9 durante las primeras 24 horas $95 \%$

29 Intervención quirúrgica en el TCE con hematoma epidural (HED) y/o subdural (HSD) 100\%

30 Utilización de corticoides en el traumatismo craneoencefálico $0 \%$

31 Incidencia de síndrome distrés respiratorio del adulto (SDRA) en el traumatismo grave $10 \%$

32 Monitorización de la presión intracraneal en el TCE grave con TAC patológica 95\%

33 Mortalidad en el traumatismo craneoencefálico grave $50 \%$

34 Osteosíntesis precoz; fracturas diafisiarias del fémur $95 \%$

35 Fijación quirúrgica precoz de las fracturas abiertas 95\%

36 Arteriografía cerebral precoz en la hemorragia subaracnoidea $90 \%$

37 Administración de nimodipino en la hemorragia subaracnoidea 100\%

38 Polineuropatía en el enfermo crítico

39 TAC inmediata en el ictus isquémico

Inferior al $50 \%$

40 Fibrinólisis intravenosa en el ictus isquémico agudo

41 Uso de los potenciales evocados somatosensoriales en la encefalopatía postanóxica

Enfermedades infecciosas

42 Bacteriemia relacionada con catéter venoso central (CVC)

4 episodios / 1.000 días de

CVC

43 Infección del tracto urinario relacionada con sonda uretral

6

episodios/1.0

00 días de sondaje

44 Neumonía asociada a ventilación mecánica (VM)

episodios/1.

000 días de

VM

50 Inicio precoz de antibioterapia en la sepsis grave

Metabolismo y nutrición

51 Complicaciones de la nutrición parenteral total (NPT): hiperglucemia y disfunción hepática Hiperglucemia:

52 Mantenimiento de niveles apropiados de glucemia

Hipoglucemia grave

Identificación de enfermos en situación de riesgo nutricional

Valoración del estado nutricional

Nutrición enteral precoz

Monitorización de la nutrición enteral

58 Requerimientos calórico-proteicos

Fuente: (Martín, y otros, 2008).

\section{Continuación tabla 2.}




\section{Generalidades de la medicina crítica o intensivista}

Vol. 3, núm. 2., (2019)

Celia María Pulgarin Fernández

\begin{tabular}{|c|c|c|}
\hline \multicolumn{3}{|c|}{ Nefrológicos } \\
\hline 60 & Indicación de depuración extracorpórea continua & $80-90 \%$ \\
\hline 61 & Uso de dopamina en el fallo renal agudo & $0 \%$ \\
\hline 62 & Incidencia de fallo renal agudo en enfermos críticos no coronarios & $10 \%$ \\
\hline 63 & Incidencia de fallo renal agudo en enfermos coronarios & $5 \%$ \\
\hline 64 & Prevención de la nefrotoxicidad inducida por contraste en la coronariografía & $90 \%$ \\
\hline 65 & Valoración del fallo renal agudo en enfermos críticos & $100 \%$ \\
\hline \multicolumn{3}{|c|}{ Sedación y analgesia } \\
\hline 66 & Monitorización de la sedación & $95 \%$ \\
\hline 67 & Sedación adecuada & $85 \%$ \\
\hline 68 & Interrupción diaria de sedación & $80 \%$ \\
\hline 69 & Manejo de la analgesia en el enfermo no sedado & $100 \%$ \\
\hline 70 & Manejo de la analgesia en el enfermo ventilado & $100 \%$ \\
\hline 71 & Uso inadecuado de la relajación muscular & $2 \%$ \\
\hline 72 & Monitorización del bloqueo neuromuscular & $100 \%$ \\
\hline 73 & Identificación del delirio & $90 \%$ \\
\hline \multicolumn{3}{|c|}{ Hemoderivados } \\
\hline 74 & Consentimiento informado en la trasfusión de componentes sanguíneos & $95 \%$ \\
\hline 75 & Trasfusión inadecuada de plasma fresco congelado & $0 \%$ \\
\hline 76 & Trasfusión inadecuada de concentrado de plaquetas & $0 \%$ \\
\hline 77 & Trasfusión inadecuada de concentrado de hematíes & $5 \%$ \\
\hline \multicolumn{3}{|c|}{ Toxicología } \\
\hline 78 & Descontaminación digestiva adecuada en intoxicaciones por vía oral & $95 \%$ \\
\hline 79 & Botiquín mínimo de antídotos en el hospital & $95 \%$ \\
\hline 80 & Hemodiálisis precoz en la intoxicación aguda & $90 \%$ \\
\hline \multicolumn{3}{|c|}{ Trasplantes } \\
\hline 81 & Donantes reales & $60 \%$ \\
\hline 82 & Valoración de trasplante hepático en la insuficiencia hepática aguda grave & $95 \%$ \\
\hline 83 & Monitorización de los donantes potenciales de órganos & $100 \%$ \\
\hline 84 & Diagnóstico de muerte encefálica & $5-30 \%$ \\
\hline \multicolumn{3}{|c|}{ Enfermería } \\
\hline 85 & Retirada de sonda nasogástrica por obstrucción & $4 \%$ \\
\hline 86 & Aspiraciones bronquiales adecuadas & $100 \%$ \\
\hline 87 & Información de enfermería a los familiares & $95 \%$ \\
\hline 88 & Traslado intrahospitalario asistido & $15 \%$ \\
\hline 89 & Presión del balón de neumotaponamiento & $95 \%$ \\
\hline 90 & Manejo de las alarmas de monitorización & $5 \%$ \\
\hline 91 & Caídas accidentales & $0 \%$ \\
\hline 92 & Cumplimentación de los registros de enfermería en el SMI & $100 \%$ \\
\hline 93 & Errores de medicación en el SMI & $5 \%$ \\
\hline 94 & Cumplimentación del protocolo de lavado de manos & $90 \%$ \\
\hline 95 & Retirada accidental de catéteres vasculares & Catéter \\
\hline & & arterial: \\
\hline & & 20 \\
\hline & & CVC: 6 \\
\hline 96 & Revisión del carro de paros & $100 \%$ \\
\hline \multicolumn{3}{|c|}{ Bioética } \\
\hline 97 & Adecuación de los cuidados al final de la vida & $100 \%$ \\
\hline 98 & Información a los familiares de los enfermos en el SMI & $100 \%$ \\
\hline 99 & Incorporación de las instrucciones previas en la toma de decisiones & $100 \%$ \\
\hline 100 & Cumplimentación del "documento de consentimiento informado" & $100 \%$ \\
\hline 101 & Limitación del esfuerzo terapéutico & $100 \%$ \\
\hline 102 & Uso de medidas de contención & $100 \%$ \\
\hline \multicolumn{3}{|c|}{ Planificación, organización y gestión } \\
\hline 103 & Existencia de un equipo de emergencias médicas en el SMI & $100 \%$ \\
\hline 104 & Suspensión de cirugía programada & $10 \%$ \\
\hline 105 & Encuesta de calidad percibida al alta del SMI & $80 \%$ \\
\hline 106 & Alta precoz o inadecuada del SMI & $1 \%$ \\
\hline 107 & Codificación al alta del SMI & $100 \%$ \\
\hline 108 & Retraso al alta del SMI & $9 \%$ \\
\hline 109 & Demora de ingreso en el SMI & $5 \%$ \\
\hline 110 & $\begin{array}{l}\text { Razón de mortalidad estandarizada } \\
0,10)\end{array}$ & Tasa: $1( \pm$ \\
\hline 111 & Tasa de necropsias & $10 \%$ \\
\hline
\end{tabular}




\section{Generalidades de la medicina crítica o intensivista}

Vol. 3, núm. 2., (2019)

Celia María Pulgarin Fernández

$\begin{array}{clc}112 & \text { Plan de acogida (PA) al profesional del SMI } & 100 \% \\ 113 & \text { Presencia de intensivista en el SMI las 24 horas del día } & 0 \%(\sin \\ & \text { intensivista) } & 100 \% \\ 114 & \text { Registro de efectos adversos } & 4 \% \\ & \text { Reingresos no programados en el SMI } & \\ \end{array}$

Fuente: (Martín, y otros, 2008).

\section{Continuación tabla 2.}

\begin{tabular}{|c|c|c|}
\hline $\mathrm{N}^{\mathrm{o}}$ & Nombre del indicador & Estándar \\
\hline \multicolumn{3}{|c|}{ Internet } \\
\hline 116 & Acceso a fuentes médicas relevantes en formato electrónico & $100 \%$ \\
\hline \multicolumn{3}{|c|}{ Formación continuada, docencia e investigación } \\
\hline 117 & Existencia de protocolos básicos & $100 \%$ \\
\hline 118 & Actividad de investigación & 1 beca/año \\
\hline 119 & Publicaciones científicas del SMI & 2 publicaciones/año \\
\hline 120 & Formación continuada & 3 créditos/año \\
\hline
\end{tabular}

Fuente: (Martín, y otros, 2008).

De los 120 indicadores (Tabla 1) definitivos se eligieron por consenso un total de 20, que fueron considerados como más relevantes o básicos para la especialidad y que la SEMICYUC entiende como de aplicación recomendable en todos los SMI. En función del tipo de indicador, la mayoría se clasificaron como indicadores de proceso (79), seguidos de indicadores de resultado (36), y de estructura (5). Las principales dimensiones de calidad evaluadas en los indicadores fueron: riesgo (54); efectividad (41); adecuación (16); satisfacción (5); eficiencia (2); continuidad asistencial (1), y accesibilidad (1). La mayoría de los indicadores valoran más de una dimensión de la calidad. Así, como ejemplo, el indicador «presencia de un intensivista en el SMI las 24 horas del día» incluiría las dimensiones de adecuación, riesgo y eficiencia (Martín, y otros, 2008).

\section{Elementos de Bioseguridad}

Con el objetivo de la contención del riesgo biológico la Seguridad Biológica dispone de los siguientes elementos básicos: 


\section{Generalidades de la medicina crítica o intensivista}

Vol. 3, núm. 2., (2019)

Celia María Pulgarin Fernández

- Prácticas de trabajo. Las personas que por motivos de su actividad laboral están en contacto, más o menos directo, con materiales infectados o agentes infecciosos, deben ser conscientes de los riesgos potenciales que su trabajo encierra y además han de recibir la formación adecuada en las técnicas requeridas para que el manejo de esos materiales biológicos les resulte seguro.

- Equipo de seguridad (o barreras primarias). Se incluyen los dispositivos o aparatos que garantizan la seguridad de un proceso (cabinas de seguridad) y los denominados equipos de protección personal (guantes, calzado, pantallas faciales, gafas, mascarillas).

- Diseño y construcción de la instalación (o barreras secundarias). La magnitud de las barreras secundarias dependerá del agente infeccioso en cuestión y de las manipulaciones que con él se realicen. Vendrá determinada por la evaluación de riesgos (Águila, 2015).

\section{Imagen I. Niveles de seguridad biológica según estándares internacionales}

\begin{tabular}{|c|c|c|c|c|}
\hline $\begin{array}{l}\text { Niveles de } \\
\text { bioseguridad }\end{array}$ & Agentes infecciosos & Pràcticas de trabajo & Barreras primarias & $\begin{array}{l}\text { Barreras } \\
\text { secundarias }\end{array}$ \\
\hline Nivel 1 & $\begin{array}{l}\text { No causan } \\
\text { enfermedad }\end{array}$ & Estándares & No requeridas & $\begin{array}{l}\text { Lavabo con agua } \\
\text { corriente }\end{array}$ \\
\hline Nivel 2 & $\begin{array}{l}\text { Peligro de infección } \\
\text { por heridas, } \\
\text { ingestión o } \\
\text { mucosas. }\end{array}$ & $\begin{array}{l}\text { Señalización. } \\
\text { Manual de } \\
\text { bioseguridad. } \\
\text { Descontaminación } \\
\text { rutinaria }\end{array}$ & $\begin{array}{l}\text { Gabinete de } \\
\text { seguridad clase I } \\
\text { oll. } \\
\text { Guantes y } \\
\text { mascarilla. }\end{array}$ & Autoclave \\
\hline Nivel 3 & $\begin{array}{l}\text { Causales de } \\
\text { enfermedades } \\
\text { serias o letales }\end{array}$ & $\begin{array}{l}\text { Todos los anteriores } \\
\text { más acceso } \\
\text { controlado. } \\
\text { Descontaminación } \\
\text { de todos los } \\
\text { desechos. } \\
\text { Descontaminación } \\
\text { de ropa de trabajo. } \\
\text { Controles } \\
\text { periódicos. }\end{array}$ & $\begin{array}{l}\text { Todos los anteriores } \\
\text { más respirador } \\
\text { autónomo cuando } \\
\text { se requiera. }\end{array}$ & $\begin{array}{l}\text { Todos los anteriores } \\
\text { más separación } \\
\text { física. } \\
\text { Puertas dobles con } \\
\text { cierre automático. } \\
\text { No recirculación de } \\
\text { aire. } \\
\text { Flujo de presión } \\
\text { negativa. }\end{array}$ \\
\hline Nivel 4 & $\begin{array}{l}\text { Alto riesgo de } \\
\text { enfermedad letal. } \\
\text { Infecciones } \\
\text { trasmitidas por aire } \\
\text { o vías desconocidas. }\end{array}$ & $\begin{array}{l}\text { Cambio de ropa } \\
\text { previo. } \\
\text { Ducha descontami- } \\
\text { nante al concluir. } \\
\text { Descontaminación } \\
\text { de todos los medios. }\end{array}$ & $\begin{array}{l}\text { Todos los anteriores } \\
\text { más trabajo en } \\
\text { gabinetes clase } \\
\text { III o uso de trajes } \\
\text { completos con } \\
\text { presión positiva } \\
\text { cuando se trabaja } \\
\text { en gabinetes clase } \\
\text { I o ll. }\end{array}$ & $\begin{array}{l}\text { Todos los anteriores } \\
\text { más edificio aislado } \\
\text { o zona caliente. } \\
\text { Sistema de } \\
\text { circulación de aire, } \\
\text { vacío y descontami- } \\
\text { nación dedicados. }\end{array}$ \\
\hline
\end{tabular}

Fuente: (Águila, 2015) 


\section{Generalidades de la medicina crítica o intensivista}

Vol. 3, núm. 2., (2019)

Celia María Pulgarin Fernández

Medidas de bioseguridad para el área de medicina intensiva y emergencias

- Utilizar los medios de protección personal (bata, gorro tapabocas y gafas) para la realización de procedimientos invasivos.

- Utilice sistema cerrado para aspiración de secreciones y líquidos orgánicos.

- Cambiar oportunamente los recipientes de los sistemas de aspiración.

- Disposición final adecuada de la ropa utilizada en los procedimientos. Enviar las muestras a los laboratorios en los recipientes adecuados.

- Disponer el material contaminado (guantes, material de curación, instrumentos) en recipientes o bolsas adecuadas para su descontaminación y disposición fi nal o recuperación.

- Efectuar desinfección y limpieza de las áreas empleando las técnicas y medios de acuerdo a los protocolos del servicio.

Las medidas enumeradas anteriormente tienen por objetivo evitar enfermedades trasmisibles por el riesgo de contaminación propiciado por el contacto directo con fluidos de pacientes; no solamente la contaminación e infección del personal sanitario, sino las infecciones cruzadas entre pacientes hospitalizados y a otras personas.

En algunas ocasiones, determinado por la actuación rápida en una emergencia, se realizan intervenciones sin la utilización de medios de protección. Se debe tener presente que siempre será posible cumplir con las medidas de bioseguridad. Es un deber nuestro para evitar daños que en ocasiones son irreparables (Águila, 2015). 


\section{Generalidades de la medicina crítica o intensivista}

Vol. 3, núm. 2., (2019)

Celia María Pulgarin Fernández

\section{Conclusiones.}

Por lo que se ha podido evidenciar en esta investigación, la medicina critica o intensiva es un área extremadamente delicada, son múltiples los factores que se relacionan ya sean externos o internos que pueden complicar la estancia de un paciente en UCI. Los controles que se deben empelar para evitar, infecciones u otras enfermedades deben ser estrictas y el personal que atiende de primera mano a los pacientes críticos deben estar altamente capacitados para prestar dichas labores.

Lo importante del avance de la medicina, es que ha podido proveer herramientas importantes para clasificar todos los elementos que se pueden presentar a la hora de establecer cuidados de pacientes críticos, hay protocolos contra infecciones, manuales de cómo se deben manejar tanto el personal de enfermería como médicos e incluso familiares de pacientes.

Debe haber un monitoreo constante tanto de los protocolos de seguridad, médicos e higiene dentro de los centros asistenciales y por supuesto dentro de las áreas críticas (UCI, sistemas de Monitoreo Intensivo, Terapia Intensiva) para corregir fallas que se puedan presentar, la final lo que cuenta es proporcionarle al paciente un cuidado de calidad que le permita una recuperación exitosa.

\section{Bibliografía}

Águila, H. R. (2015). Medicina Intensiva y Emergencias. Manual de Procedimientos Invasivos En Medicina Intensiva Y Energencia, 135-138.

Briceño, I. (2005). Sepsis: Definiciones y aspectos fisiopatológicos. Medicrit, 2(8), 164-178.

Luna, C., Gherardi, C., Famiglietti, A., \& Vay, C. (2001). Resistencia bacteriana y antibioticoterapia en medicina respiratoria y terapia intensiva. Medicina, 61(5), , 603-13. 


\section{Generalidades de la medicina crítica o intensivista}

Vol. 3, núm. 2., (2019)

Celia María Pulgarin Fernández

Martín, M. C., Cabré, L., Ruiz, J., Blanch, L., Blanco, J., Castillo, F., \& Saura, R. (2008). Indicadores de calidad en el enfermo crítico. Medicina intensiva, 32(1), 23-32.

Padilla-Cuadra, J. I. (2018). Medicina evolucionista en terapia intensiva: un nuevo paradigma. Acta Médica Costarricense, 60(1), 7-14.

Palencia Herrejón, E., González Díaz, G., \& Mancebo Cortés, J. (2011). El futuro de la Medicina Intensiva. Medicina Intensiva, 35(4), 232-235.

Pittet, D., \& Harbarth, S. (2006). El control de la infección en Terapia Intensiva. Medicina Intensiva.

Vera Carrasco, O. (2012). Programas académicos de la residencia médica en medicina crítica y terapia intensiva. Revista Médica La Paz, 18(2), 70-86.

Vera Carrasco, O. (2015). Origen y desarrollo histórico de la Medicina Crítica y Unidades de Cuidados Intensivos en Bolivia. Revista Médica La Paz, 21(2), 77-90.

Zaragoza Crespo, R., \& Palomar Martínez, M. (2011). Puesta al día de medicina intensiva sobre el enfermo crítico con infección grave: ¿qué hemos aprendido? Medicina Intensiva, 35(3), 186-188.

$$
\text { (9) (1) } \Theta(0
$$

\section{RECONOCIMIENTO-NOCOMERCIAL-COMPARTIRIGUAL}

CC BY-NC-SA

ESTA LICENCIA PERMITE A OTROS ENTREMEZCLAR, AJUSTAR Y CONSTRUIR A PARTIR DE SU OBRA CON FINES NO COMERCIALES, SIEMPRE Y CUANDO LE RECONOZCAN LA AUTORÍA Y SUS NUEVAS CREACIONES ESTÉN BAJO UNA LICENCIA CON LOS MISMOS TÉRMINOS. 